\title{
A STUDY ON ROLE OF SMART PHONE APPLICATIONS IN IMPLEMENTING E-HEALTH SERVICES IN UTTARAKHAND
}

\author{
Mukesh Joshi ${ }^{1}$, Durgesh Pant ${ }^{2}$ \\ ${ }^{1}$ Research Scholar, Teerthanker Mahaveer University, Moradabad \\ ${ }^{2}$ Director School of Computer Science, Uttarakhand Open University, Haldwani
}

\begin{abstract}
There are various healthcare applications present in today's world. Most of these applications are not offering any medical or healthcare services, but they can be used as point of solutions for collecting and presenting data or address as a single aspect of health or wellness. For Uttarakhand this can be a boon as geographical hindrances are present in health sector of Uttarakhand.
\end{abstract}

Keywords: Smart Phone, EHealth, Uttarakhand, Android

\section{INTRODUCTION}

The use of health care applications through mobile/smart phones is emerged or known as mHealth. Currently mHealth applications includes the use of smart phones in collecting the data based on community, delivering of health care information to medical professional, researchers and patients as well as real time monitoring of the patients status can be done by using some of the available applications of smart phones.

Use of these smart phone applications in Uttarakhand is very helpful because of its hilly and disaster prone conditions. As the availability of wireless network is quite effectively present in Uttarakhand as per the Annual report of DoT [1]. In this research paper authors are trying to show the use of various smart phone applications which helps in improving eHealth services in Uttarakhand.

\section{ABOUT UTTARAKHAND}

The state of Uttarakhand has International borders with Nepal and Tibet from eastern and northern part respectively. Moreover, Himachal Pradesh from western part and Uttar Pradesh from southern part are surrounding the state of Uttarakhand. As per the geographical area the state is about 53,483 Sq. Km [2]. As per the Census 2011, the state population was 1.0 Crore (Approx.) Uttarakhand was carved from existing Uttar Pradesh on 9. Nov. 2000 and become the twenty seventh state of Independent India. State of Uttarakhand was divided in two parts i.e. Kumaun and Garhwal and this would enhance the pace of social and economic development of Uttarakhand population. Initially the state has been created with thirteen districts of Uttar Pradesh. At later stage it was further divided by government of in forty nine sub divisions and ninety five development blocks.

There are 15,638 inhabited villages and 86 urban settlements in the state. However, due to geographical constraints it is very tough to create policies based on health care conditions of this state as well as due to various physical, logical and environmental problems in Uttarakhand [3, 4].

\section{What are Mobile Medical Apps?}

Before beginning the discussion about the Smart phone applications [4] we must know about what we mean by mobile medical apps: "Mobile medical apps means the software programs that run on smartphones and other mobile communication devices. They can also be the accessories that may attach to a smartphone or other mobile communication devices, or a combination of accessories and software"[5].

\section{E-HEALTH APPS AND SMART PHONES}

A smartphone is a mobile phone which is based on web operating system, with more innovative computing and communication facility in comparison to any other mobile phone. In India and in its states Smart phones are becoming a more and more important platform for the distribution of health education and medical interventions [6,7]. Most of the modern generation smartphones gradually viewed as handheld computers rather than as phones, due to their powerful on-board computing-competency, capacious memories, enormous screens, on-board personal administration tools, high quality cameras and open source operating systems that instigate application (apps) development. Relatively in a short span of time, smartphone has speared there scope expressively in all around the world by capturing an entire age spectrum of subscribers from present generation. Smartphone knowledge can change the method that the medicine is learnt and experienced in past time. At present this technology can be used more likely in Uttarakhand to access appropriate, frequently updated, webbased literature than refer to hard copies of records or journals.

The most common web based mobile operating system (OS) used by recent smartphones include Android by Google's, iOS (iPhone OS) by Apple systems, Symbian Operating system Nokia and many more [8]. 
Among these, smartphones with Android and iOS of googles and Apple's respectively are very commonly used at contemporaneous world. Android based operating system is very widespread among Indian market by having a market share of 90 percent [9] in comparison to other operating system. The usage of smart mobile phone in health care services in Uttarakhand provides a vital of anytime, anywhere service into the entire state. Mobile based applications are on the escalation, with many students of medical profession, clinician and allied health workers, as they are accepting smart phone mobile health applications effectively in their practices in Uttarakhand also [10].

Some studies $[11,12]$ have assessed the use of smartphones to support healthcare and community health involvements, remarkably in the collection and comparison of data for healthcare based research, healthcare learning and clinical practice in the community of our country. These web based mobile phones can also be used to support telemedicine and distant healthcare in Uttarakhand and other part of our country. Smartphones based application can support in physical examination of patients like checking hearing problems, eyesight, and color recognition issues etc. It can also assess mental status; or photo or video documents physical findings. Patients by his own can also use these applications for retrieving their health data, making contact with their healthcare service providers and also by actively contributing in their own care i.e. participatory healthcare through application of smartphones. In Uttarakhand we can also recommend the mandatory use of smart phones to all students studying in various medical colleges.

\section{E-HEALTH APPLICATIONS FOR DOCTORS/ HEALTH PRACTITIONERS}

1. Speed Anatomy: These apps are useful for students/researchers at school or college level who are studying Anatomy. This mobile application can be used for detecting the muscle, arteries and veins strength in human body. This application could be used in trauma situation or if we are not equipped with the devices to check muscles or veins status for patients residing in remote areas of Uttarakhand where availability of resources is quite tough. This application is also known by Speed bones MD or Speed Angiology MD

2. Medical encyclopedia - Medical Encyclopedia or a Medical Library application is especially designed for medical professionals or the persons interested in medical science. It complies of a huge database consisting of thousands of articles about human diseases, test and there conditions, symptoms of various diseases etc. It can be treated as a library for medical students. This application may help in providing better services in health in remote areas of Uttarakhand as Due to various factors it is not easy for doctors to update their knowledge level. This concept can be implemented through and android application called PublicMed mobile Pro or Medscape
3. MedPage today mobile - This application can be termed as a blog based activity in medical services. In this application various specialists are sharing their views on various trends in medical services. MedPage application is especially designed for doctors and physicians or medical students can also use it for enhancing their knowledge. This also provides the breaking happenings going on medical services.

4. MedCalc - It is a statistical software package designed for the biomedical sciences. This application could be used in medical research as it allows the writing of various medical formulas in proper manner. The application can also be used for calculating results of various medical researches.

5. Epocrates - This application is used by medical practitioners dealing with drugs. It is one of the established methods for getting information about latest drugs and its dose for different age groups. The application is very useful for the patients as it provide the harms and warning regarding a specific medicine.

6. Quick LabRef- Application use to retrieve information about pathological data. It provides necessary help i.e. by providing ranges, about clinical data mentioned in the report. As one can analyse his/her pathological data by using QuickLabRef application.

7. Isabel- Application use by doctors for performing a diagnosis. In this app doctor provide the information for a particular scenario and he/she get possible diagnosis as well as medication possible for the particular situation.

8. ResolutionMD- This app allows doctors to view X- ray of a particular patient through smart phone. This helps the doctor to perform the analysis on particular X-ray images independent of their location.

9. Clinicam- This is a database based application, as it was used by doctors for capturing the patient's photographs and uploads it to the patient electronic health record directly. Through this application each information about patient is directly move to his/her health record instead of copying in local computer/phone of a doctor. This could help us in preventing cyber laws associated to health care.

10. Taber's medical dictionary - Taber's is the foremost medical dictionary used by healthcare experts. This application holds thousands of terms including their images associated with diagnosis, nursing and more.

11. Sanford guide- This is one of the best application used in Uttarakhand as it provides useful information for disaster and epidemic outbreak. This provides the resources to handle patient and there disease during epidemic outbreak.

12. Drug calc- This app could be used by the patients to take the proper dose of drug as prescribed by the doctor. By using this application weight of drugs can be taken so that exact amount of drug could be taken by the patients. 


\section{E-HEALTH APPLICATIONS FOR INDIVIDUALS/ PATIENTS}

1. My pregnancy today- This application was very useful for pregnant women residing at different locations. It can be one of the best parenting resource supporting more than twenty five million womens at present. In this application due date of delivery is entered by a pregnant women and at the same moment the smart phone is converted to expert guide. Now it provides day to day information during pregnancy as well as it provides suitable solutions for the questions raises by pregnant womens related to their pregnancy issues.

2. WomanLog Calendar- This application was used by womens during her conceive period. The application provides fertility calendar which was very helpful for womens to know about their fertility period.

3. Draw MD series- The application was used by doctors to provide better information to their patients. By using this application doctor could elaborate the surgical procedure to the patient before initiating the surgery. Some basic surgeries could be explained under this app are heart related surgery, Urine based surgery and many more. Thus, this application helps in improving the coordination among doctor and patient.

4. Radiology 2.0- This application can be act as a data ware house of radiology cases. Under this application various case studies related to radiology are present with suitable prescription that can be used by doctors/ patient as per there requirements.

5. ECG Guide- This application was used by patients to make the analysis for his/her electrocardiogram. This could be very helpful as we are totally dependent on doctors' saying regarding our ECG information.

6. Paeds ED- Application is very helpful for smart phone users. This helps us to know about the paediatric drug and its side effects based on age and situation.

7. Healthbook - This application could be our nutrition guide as it provides the nutrition details as per our fooding habits.

8. OsiriX- This application is very useful for carrying our radiological images through a handheld device.

\section{CONCLUSION}

As per the discussions with various doctors/ health professionals regarding health services in Uttarakhand most of them believed that many of these smart phone based mobile applications are benefiting out state. It helps in reducing the demand of machines and other equipment's required for providing better health care services in Uttarakhand. Effective monitoring of the patients can be done by using these applications in state of where distance and geographical conditions are the major barriers in health service implementation. Still, the field has a way to go, doctors add, particularly when it comes to making good use of all the patient data being generated.

Finally we complete the paper by suggesting that, in the initial phase most of these applications can be initiated through sub center which is present at level I in 3 tier health architecture of Uttarakhand.

\section{REFERENCES}

[1]. Report by department of telecommunications Ministry of communications \& Information technology Government of India New Delhi, 2011 (Retrieved from www.dot.gov.in on 2.May.2014)

[2]. http://uk.gov.in/home/index1 (Accessed on 1. Nov.2014)

[3]. Joshi, M., \& Durgesh, P., 2013, A study for ICT enable e-health services for Uttarakhand. Pezzottaite Journals, ISSN: 2319-9016.

[4]. e-governance roadmap (egrm) report . Retrieved from Government of Uttarakhand website :http // itda . uk . gov . in upload/contents/File-35.pdf

[5].http://www.fda.gov/MedicalDevices/ProductsandMedical Procedures/ConnectedHealth/MobileMedicalApplications/uc m255978.htm (accessed on 1.Nov.2014)

[6]. http://en.wikipedia.org/wiki/Smartphone (Accessed on 2. Aug.2014)

[7]. N Nawka, Sharma A.K., 2011, A scalable extensible smart-phone based mobile gateway and application for remote health monitoring. Published in Conference on Internet Multimedia Systems Architecture and Application (IMSAA), IEEE.

[8]. VO Alexander, Brooks G (2011), Benefits of Telemedicine in Remote Communities \& Use of Mobile and Wireless Platforms in Healthcare. Statewide Health Plan 2011-2016 report.

[9]. M omar,M Dawson, 2013, Research in Progress Defending Android Smartphones from Malware Attacks, published in Advanced Computing and Communication Technologies (ACCT), Third International Conference , IEEE.

[10]. http://timesofindia.indiatimes.com/tech/technews/Androids-market-share-in-India-over-90-

IDC/articleshow/22793647.cms Accessed on 1.May.2014)

[11]. Vaisala K.S, Sharma M.K., 2012, E-health for rural areas of Uttarakhand under e-Governance service delivery model (20,Published in International Conference on Recent Advances in Information Tecnology, RAIT, by IEEE.

[12]. Shinan Wang, Weisong Shi,Arnetz B.B, Wiholm C, SPARTAN: A framework for Smart Phone Assisted RealTime Health care Network design (2010), Published in International Conference on Collaborative Computing: Networking, Applications and Worksharing (CollaborateCom), RAIT, by IEEE. 